Corrosion Science, 2007, v.49, n.4, p.2021-2036

ISSN: 0010-938x

DOI: $10.1016 /$ j.corsci.2006.10.017

http://www.elsevier.com

http://www.elsevier.com/wps/find/journaldescription.cws_home/260/description\#description

http://www.sciencedirect.com/science?_ob=MImg\&_imagekey=B6TWS-4MKTXYF-1-

$1 \& \_c d i=5570 \& \_u s e r=3366836 \& \_p i i=S 0010938 X 06003404 \& \_$orig $=$browse\&_coverDate $=04 \% 2 F 30 \% 2 F 2007 \& \_s k$

$=999509995 \&$ view $=c \& w c h p=d G L b V l b-z S k W b \& m d 5=c a 2 f d f 542511366 c 48839 b 6 f 07$ ce66ed \&ie=/sdarticle.pdf

(C) 2006 Elsevier Ltd. All rights reserved.

\title{
Potential control under thin aqueous layers using a Kelvin Probe
}

\section{G.S. Frankel a, M. Stratmann ${ }^{\text {b }}$, M. Rohwerder ${ }^{\text {b }}$, A. Michalik ${ }^{\text {b }}$, B. Maier ${ }^{\text {a }}$, J. Dora ${ }^{\text {c }}$, M. Wicinski $^{\text {d }}$}

a Fontana Corrosion Center, Department of Materials Science and Engineering, The Ohio State University

b Max Planck Institute for Iron Research, Dusseldorf, Germany

c Dora Power Systems, Wroclaw, Poland

d K\&M SoftControl, Duesseldorf, Germany

\begin{abstract}
Kelvin Probes can be modified to control as well as monitor potential. The design and operation of two different Kelvin Probe Potentiostats (KPPs) are described in this paper. One approach uses a permanent magnet and double coil to oscillate the needle at a fixed frequency, an AC backing potential, and software analysis and control schemes. This technique can also control the distance between the tip and sample, thereby tracking the topography of the sample. Both KPPs were used to make measurements on Type 304L stainless steel under thin layers of electrolyte. Cathodic polarization curves exhibited a limiting current density associated with oxygen reduction. The limiting current density varied with solution layer thickness over a finite range of thickness. Anodic polarization curves on $304 \mathrm{~L}$ in a thin layer of chloride solution resulted in pitting corrosion. The breakdown potential did not vary with solution layer thickness. However, the thin layer was observed to increase in volume remarkably during pit growth owing to the absorption of water from the high humidity environment into the layer with ionic strength increased by the pit dissolution. The open circuit potential (OCP) and solution layer thickness were monitored during drying out of a thin electrolyte layer. Pitting corrosion initiated, as indicated by a sharp drop in the OCP, as the solution thinned and increased in concentration.
\end{abstract}

\section{Introduction}

Atmospheric corrosion occurs under thin aqueous layers that are adsorbed films in equilibrium with humid gaseous environments or thicker layers formed by precipitation or condensation during outdoor exposure. Electrochemical measurements clarifying the kinetics and mechanisms of atmospheric corrosion are complicated by problems associated with immersing a reference electrode (RE) and counter electrode (CE) into the thin layer.

Mansfeld and Kenkel described the use of atmospheric corrosion rate monitors, composed of interdigitated fingers of different metals, that track the galvanic current flowing through the cross-section of stacks of alternating dissimilar metal plates [1]. The maximum current was found to flow at the point just before complete drying owing to the increase in oxygen diffusion rate with decreasing solution layer thickness. Mansfeld used similar devices composed of a single material to make measurements of polarization resistance [2]. The measurements were two-electrode measurements with noreference electrode. Lyon and coworkers [3-7] measured electrochemical kinetics in layers as thin as $50 \mu \mathrm{m}$ using an essentially bulk electrochemistry approach involving a capillary connection to a remote RE and CE.

The problem of introducing an RE into the thin water layer responsible for atmospheric corrosion was solved by Stratmann and Streckel who demonstrated that a Kelvin Probe (KP) 
vibrating above a sample provides a measure of the corrosion potential [8-10]. They made corrosion rate measurements by monitoring the rate of decrease in the oxygen partial pressure [9]. Stratmann and Streckel also used a modification of the KP apparatus to control the potential and measure the current to a coplanar counter electrode, thereby achieving the ability to measure polarization curves [10]. This arrangement might be called a Kelvin Probe Potentiostat (KPP). They used the KPP to measure cathodic polarization curves on Pt under thin layers of $1 \mathrm{M}$ Na2SO4. The limiting current density for oxygen reduction was found to vary inversely with the thickness of the electrolyte layer for layers with thickness between about 10 and $100 \mu \mathrm{m}$. [10] Convection caused by the Kelvin Probe needle controlled the diffusion layer thickness for thick layers and transfer of oxygen across the gas/electrolyte interface was the rate limiting step for the electrolyte layers thinner than about $10 \mu \mathrm{m}$. Polarization curves on Fe under thin layers of $1 \mathrm{M}$ $\mathrm{Na} 2 \mathrm{SO} 4$ revealed that the slowing of metal dissolution in thin layers counteracted the enhancement of oxygen reduction. The corrosion rate was found to reach a maximum at about 10 $\mu \mathrm{m}$ and then decrease as the layer thickness decreased further [10].

Electrochemical measurements under thin electrolyte layers were also reported by Nishikata, Tsuru and coworkers in a series of papers [11-16]. They first performed Electrochemical Impedance Spectroscopy (EIS) measurements on samples composed of two identical electrodes of steel, stainless steel, or $\mathrm{Cu}$ embedded in epoxy under thin electrolyte layers during cyclic wet-dry conditions $[11,12]$. They subsequently developed a three-electrode approach using a coplanar chloridized Ag RE embedded in epoxy between the Fe working electrode (WE) and the CE [13]. The RE allowed for the measurement of cathodic polarization curves, and they found the same dependence of limiting current density on layer thickness as did Stratmann et al. The corrosion rate was found to be maximal at a layer thickness of 20-30 $\mu \mathrm{m}$, and $\mathrm{pH}$ had an influence only in thick layers.

Nishikata, Tsuru and coworkers also studied pitting corrosion of stainless steel under thin electrolyte layers [14-16]. EIS measurements were made using a coplanar chloridized Ag RE as described above. The corrosion rate associated with pitting was assessed by EIS during cyclic wet-dry cycling on different stainless steels [14]. Corrosion potential measurements were made on a second embedded working electrode using yet another sample design in which an agar-filled hole in the epoxy allowed ionic connection to a remote standard RE [15]. A decrease in the corrosion potential by hundreds of $\mathrm{mV}$ coincided with a sharp decrease in polarization resistance and the onset of pitting corrosion as a critical chloride concentration was reached during drying [15]. A thin electrolyte layer was found to encourage pit growth by reducing convection and thereby maintaining a concentrated electrolyte within the pit. In a recent study, the critical concentration for initiation of pitting corrosion of stainless steel in a thin electrolyte during drying at various fixed rates was determined [16]. The coplanar $\mathrm{Ag} / \mathrm{AgCl} \mathrm{RE}$ and agar-filled hole used in the work of Tsuru et al. suffer from problems associated with changing $\mathrm{Cl}$ concentration and nonuniform current distribution.

In this study, the use of KPPs for the study of electrochemical reactions electrodes under thin electrolyte layers is revisited. Two different KPP instruments are described and then results from the different instruments on Type 304 SS are presented and discussed.

\section{Instrumentation}

In this section, two different KP instruments will be described. Both have the appropriate translation stages and software to allow them to be used as scanning Kelvin Probes, but have not been operated in that mode when used as KPPs. 


\subsection{Design A}

Fig. 1 shows a schematic of a KP that was modified to operate as a KPP. The $1 \mathrm{~mm}$ diameter wire $\mathrm{WE}$ and a concentric, coplanar ring $\mathrm{CE}$ are embedded in epoxy, resulting in a configuration similar to a ring-disk electrode. More detail on this assembly is given below. Vibrating directly above the WE is the Type 304 stainless steel KP needle, which has a diameter equal to that of the WE and acts as an RE in the electrochemical circuit in KPP mode. The potential of the needle is calibrated above a small $\mathrm{Cu}$ boat containing saturated $\mathrm{CuS} 04$. The needle is vibrated in near resonance by a small speaker coil and membrane, which is a low power and low noise method to excite the needle. In this setup, unlike previous KP designs $[8-10,17]$ the WE is grounded and the needle is not. This configuration allows the WE to be connected to other grounded devices such as meters and standard potentiostats. As a result, the needle must be electrically isolated from the rest of the vibration setup. Insulating epoxy is used to connect it to another needle that fits into a socket on the membrane surface, and a very fine wire is used to connect the KP needle to the system circuitry.

The KP signal is directed into an operational amplifier and then into a lock-in amplifier that monitors the signal at the oscillation frequency. The output of the lock-in goes then to an integrator, and the integrator output into a switch box. The position of the switch box in Fig. 1 is set for normal KP operation with no potential control. As shown in the figure, in normal mode the integrator output is directed to the other input of the op-amp, opposite the KP signal. In this design, the integrated signal will change until it equals the KP signal, at which point it equals the contact potential difference, VCPD. This technique is therefore not a direct nulling method whereby the KP signal is nulled. Rather, the KP signal is measured by nulling the output of the op-amp. Note that there is a switch in the integrator circuit that allows for manual rather than automatic nulling. Manual nulling is typically used as a diagnostic tool and the KP is usually operated in automatic mode.

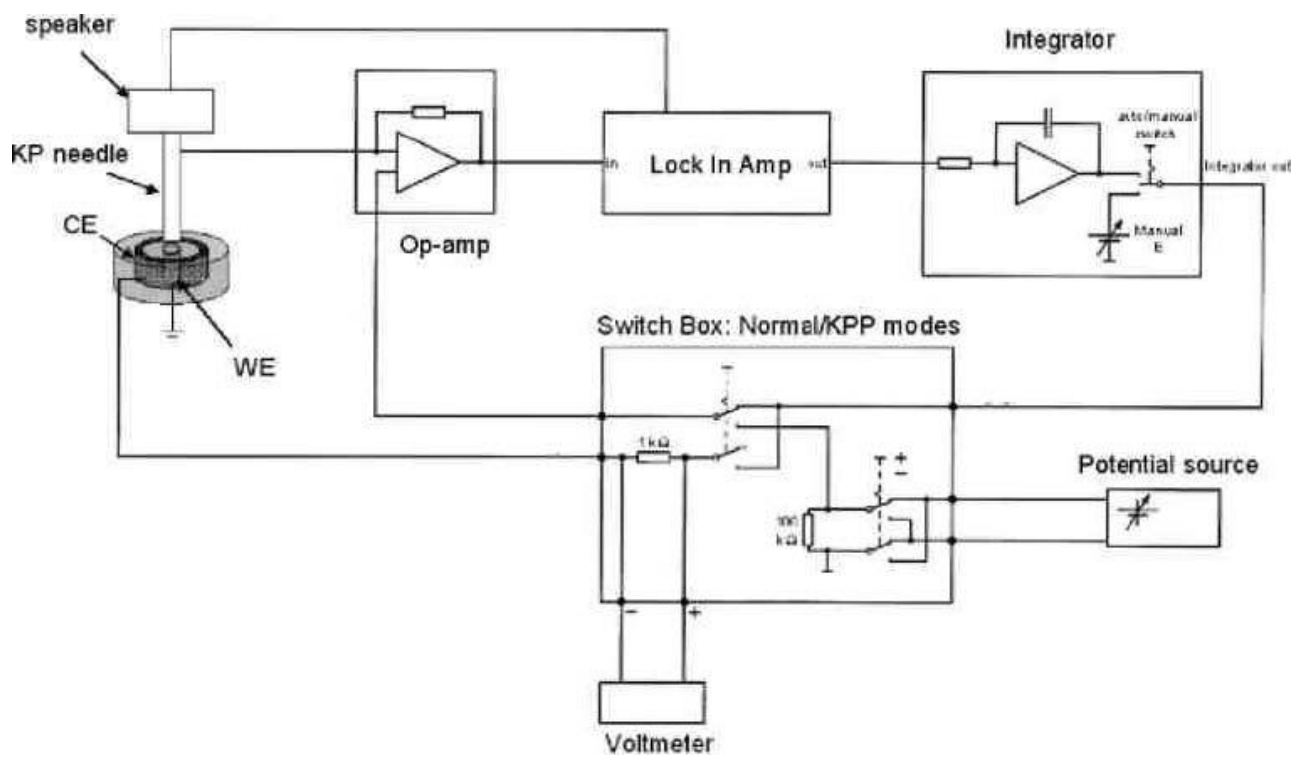

Fig. 1. Schematic diagram of KP and KPP according to Design A. Switch box set to normal KP mode.

The circuit described above was modified to provide potential control capability by means of the switch box shown in Fig. 1. In the potential control mode, the integrator switch is set to manual mode and the switch box to KPP mode. A control potential from a voltage source 
is inputted into the op-amp rather than the integrator output, which is instead redirected to the $\mathrm{CE}$. With this configuration, the current to the CE, which flows through the thin film electrolyte to the grounded WE, is varied until the measured KP signal equals the set potential supplied by the voltage source. This is analogous to the operation philosophy of a normal potentiostat. A software program is used to set endpoint potentials, potential step size and duration. The current is monitored at $3 \mathrm{~Hz}$, and the last $10 \%$ of the potential values in each step are averaged to provide a value at a given potential.

This KPP was successfully used to measure anodic and cathodic polarization curves under thin films of electrolyte, as described below. However, there are problems with this design that can create operational difficulties. A nulling technique is by nature low signal-to-noise. KPs can operate off-null with higher signal-to-noise and then determine the null condition by interpolation or extrapolation [18]. Another problem relates to the excitation of the needle assembly at resonance. The resonance condition sometimes can be difficult to find, and easy to lose. In other words, small changes in the vibrating system can create a change in the resonant frequency, which would then change the amplitude of vibration at a fixed frequency and thus affect the KP signal. Finally, the grounded sample eliminates problems associated with isolating the sample from the grounded chamber in an environment with high relative humidity. However, it then becomes possible to pick up a signal from all of the grounded components such as the chamber walls.

\subsection{Design $B$}

The second KPP design utilizes a very different approach in an effort to address the shortcomings of design A listed above. Fig. 2 shows a schematic diagram of the design B KPP. The left part of the figure reflects the components involved in potential measurement, and the right part is the electrochemical control circuitry. The WE/CE assembly and vibrating KP needle are identical to design A. However, in this design, the KP needle is attached to a permanent magnet, which is vibrated at a fixed frequency, approximately $1 \mathrm{kHz}$, using a double coil. It is critical in this design to shield this magnetic field effectively to prevent interaction with the measurement. However, with proper shielding, the vibration frequency is well controlled and constant, in contrast to the resonance condition of design A.

An off-null approach is taken for measurement of contact potential difference in design B. A sinusoidal potential of frequency $10 \mathrm{~Hz}$, the so-called backing potential, is applied between the $\mathrm{WE}$ and ground. The resulting $\mathrm{KP}$ current, $i_{\mathrm{KP}}$, is

$$
i_{\mathrm{KP}}=\frac{\mathrm{d} Q}{\mathrm{~d} t}=\frac{\mathrm{d}(V \cdot C)}{\mathrm{d} t}=\frac{\mathrm{d} V}{\mathrm{~d} t} \cdot C+V \cdot \frac{\mathrm{d} C}{\mathrm{~d} t}
$$

where $\mathrm{Q}$ is charge, and $\mathrm{V}$ and $\mathrm{C}$ are the voltage and capacitance between the sample and needle,

respectively. Since $C=\frac{\varepsilon \varepsilon_{0} A}{d}=\frac{\varepsilon \varepsilon_{0} A}{d_{0}+\Delta d \sin \left(\omega_{1} t\right)}$ and $V=V_{3} \sin \left(\omega_{2} t\right)+V_{C P D}$,

$$
i_{K P}=C V_{B} \omega_{2} \cos \left(\omega_{2} \cdot t\right)+\left(V_{B} \cdot \sin \left(\omega_{2} \cdot t\right)+V_{C P D}\right)\left(C_{0} \omega_{1} \frac{\Delta d}{d_{0}} \cos \left(\omega_{1} t\right)\right)
$$

where $\varepsilon$ is the dielectric constant, $\varepsilon 0$ is the permittivity of free space, $A$ is area, $d$ is separation distance between the sample and needle, $d 0$ and $\Delta d$ are the average separation distance and needle vibration amplitude, respectively, $V B \sin (\omega 2 t)$ is the backing potential with $\omega 2=10 \mathrm{~Hz}$, 
VCPD is the contact potential difference, $\mathrm{C} 0$ is the average capacitance, and $\omega 1=1 \mathrm{kHz}$ is the needle vibration frequency. Since $(\Delta d / d 0) \cdot \omega 1 \gg \omega 2$, the first term can be neglected if the vibration amplitude is not too small, otherwise the signal will be determined by the $\omega 2$ term. The resulting Kelvin Probe current and potential measured using a high impedance meter with input impedance $R$ are thus modulations of the two frequencies:

$$
\begin{aligned}
& i_{\mathrm{KP}}=C_{0} \cdot\left(V_{\mathrm{B}} \sin \left(\omega_{2} \cdot t\right)+V_{\mathrm{CPD}}\right) \cdot \frac{\Delta d}{d_{0}} \cdot \omega_{1} \cos \left(\omega_{1} \cdot t\right) \\
& V_{\mathrm{KP}}=R C_{0} \cdot\left(V_{\mathrm{B}} \cdot \sin \left(\omega_{2} \cdot t\right)+V_{\mathrm{CPD}}\right) \cdot \frac{\Delta d}{d_{0}} \cdot \omega_{1} \cos \left(\omega_{1} \cdot t\right)
\end{aligned}
$$

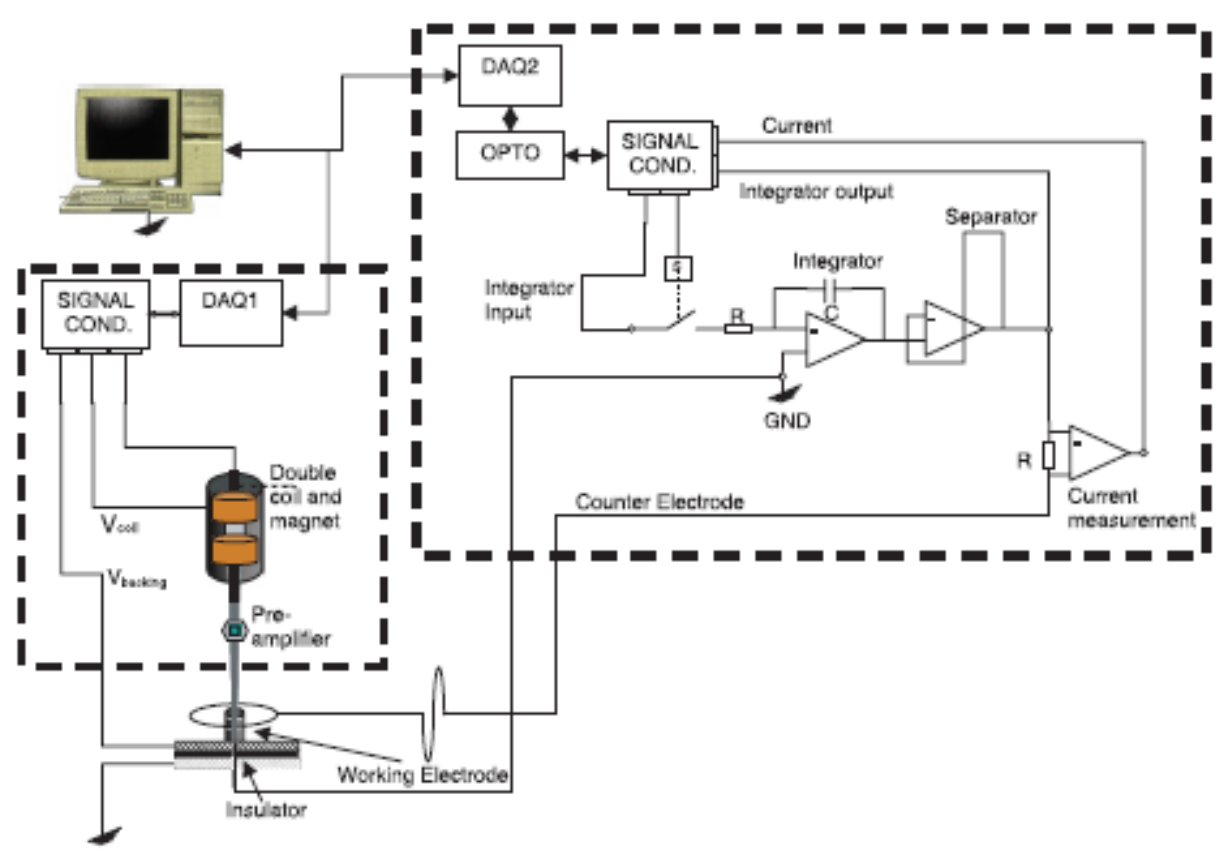

Fig. 2. Schematic diagram of KP and KPP according to Design B. Dashed box on left represents potential measurement system. Dashed box on right represents potential control system.

In a standard nulling approach, a DC backing potential is added to the circuit and is equal to -VCPD when the KP signal is nulled. The use of an AC backing potential in design $\mathrm{B}$ results in a KP signal, given by Eq. (3), that depends on the polarity of $V C P D$ and the relative magnitude of $V C P D$ and $V B$. There are four different cases to consider:

Case 1: $V C P D>0$ and $|V C P D|<|V B|$

Case 2: $V C P D<0$ and $|V C P D|<|V B|$

Case 3: $V C P D>0$ and $|V C P D|>|V B|$

Case 4: $V C P D<0$ and $|V C P D|>|V B|$

The form of VKP is different for the four cases, as shown in Fig. 3. For all cases, VKP is a modulated signal, combining the frequencies of vibration and backing potential. The signal is collected by the computer and further processed by determining absolute value, applying a peak finder routine, and then smoothing, to get the responses shown on the right side of Fig. 3. The form of the processed signal is different for each case. For Cases 1 and 2, two maxima are observed, and for Cases 3 and 4, a maximum and a minimum are observed. The values of the 
two maxima or the maximum and minimum depend on a scaling factor, $k$, related to the needle/electrolyte separation distance, $V C P D$ and $V \mathrm{~B}$ according to the following equations for Cases 1-4, respectively:

$$
\begin{aligned}
& \text { Case } 1: k \cdot(\max 1(V \mathrm{KP})+\max 2(V \mathrm{KP}))=|V \mathrm{~B}+V \mathrm{CPD}|+|-V \mathrm{~B}+V \mathrm{CPD}|=2 V \mathrm{~B} \\
& \text { Case } 2: k \cdot(\max 1(V \mathrm{KP})+\max 2(V \mathrm{KP}))=|V \mathrm{~B}-V \mathrm{CPD}|+|-V \mathrm{~B}-V \mathrm{CPD}|=2 V \mathrm{~B} \\
& \text { Case } 3: k \cdot(\max (V \mathrm{KP})-\min (V \mathrm{KP}))=|V \mathrm{~B}+V \mathrm{CPD}|-|-V \mathrm{~B}+V \mathrm{CPD}|=2 V \mathrm{~B} \\
& \text { Case } 4: k \cdot(\max (V \mathrm{KP})-\min (V \mathrm{KP}))=|V \mathrm{~B}-V \mathrm{CPD}|-|-V \mathrm{~B}-V \mathrm{CPD}|=2 V \mathrm{~B}
\end{aligned}
$$

After the needle/electrolyte distance is found, the scale factor $k$ is calculated only once from one of the above equations using the values of the two maxima or the maximum and minimum in the process signal. As an example, for Case 1:

$$
k=\frac{2 V_{\mathrm{B}}}{\max _{1}\left(V_{\mathrm{KP}}\right)+\max _{2}\left(V_{\mathrm{KP}}\right)}
$$

This scaling factor is then kept constant as a means of height control. This also provides data that can be used for topographic mapping. It is now possible to determine VCPD, which is performed again from the values of the maxima or the maximum and minimum according to the following equations for the four cases:

Case $1: k \cdot(\max 1(V \mathrm{KP})-\max 2(V \mathrm{KP}))=|V \mathrm{~B}+V \mathrm{CPD}|-|-V \mathrm{~B}+V \mathrm{CPD}|=2 V \mathrm{CPD}$

Case $2: k \cdot(\max 1(V \mathrm{KP})-\max 2(V \mathrm{KP}))=|V \mathrm{~B}-V \mathrm{CPD}|-|-V \mathrm{~B}-V \mathrm{CPD}|=2 V \mathrm{CPD}$

Case $3: k \cdot(\max (V \mathrm{KP})+\min (V \mathrm{KP}))=|V \mathrm{~B}+V \mathrm{CPD}|+|-V \mathrm{~B}+V \mathrm{CPD}|=2 V \mathrm{CPD}$

Case $4: k \cdot(\max (V \mathrm{KP})+\min (V \mathrm{KP}))=|V \mathrm{~B}-V \mathrm{CPD}|+|-V \mathrm{~B}-V \mathrm{CPD}|=2 V \mathrm{CPD}$

As an example, again for Case 1:

$$
V_{C P D}=\frac{k\left(\max _{1}\left(V_{K P}\right)-\max _{2}\left(V_{K P}\right)\right)}{2}=V_{B} \frac{\left(\max _{1}\left(V_{K P}\right)-\max _{2}\left(V_{K P}\right)\right)}{\left(\max _{1}\left(V_{K P}\right)+\max _{2}\left(V_{K P}\right)\right)}
$$

During the measurement, a DC offset is added to the backing potential, $v B=(V D C+V B \sin (\omega 2 \cdot$ t)), to make the magnitude of the two peaks in the processed signal similar, which improves the reproducibility and precision of the measurement. 

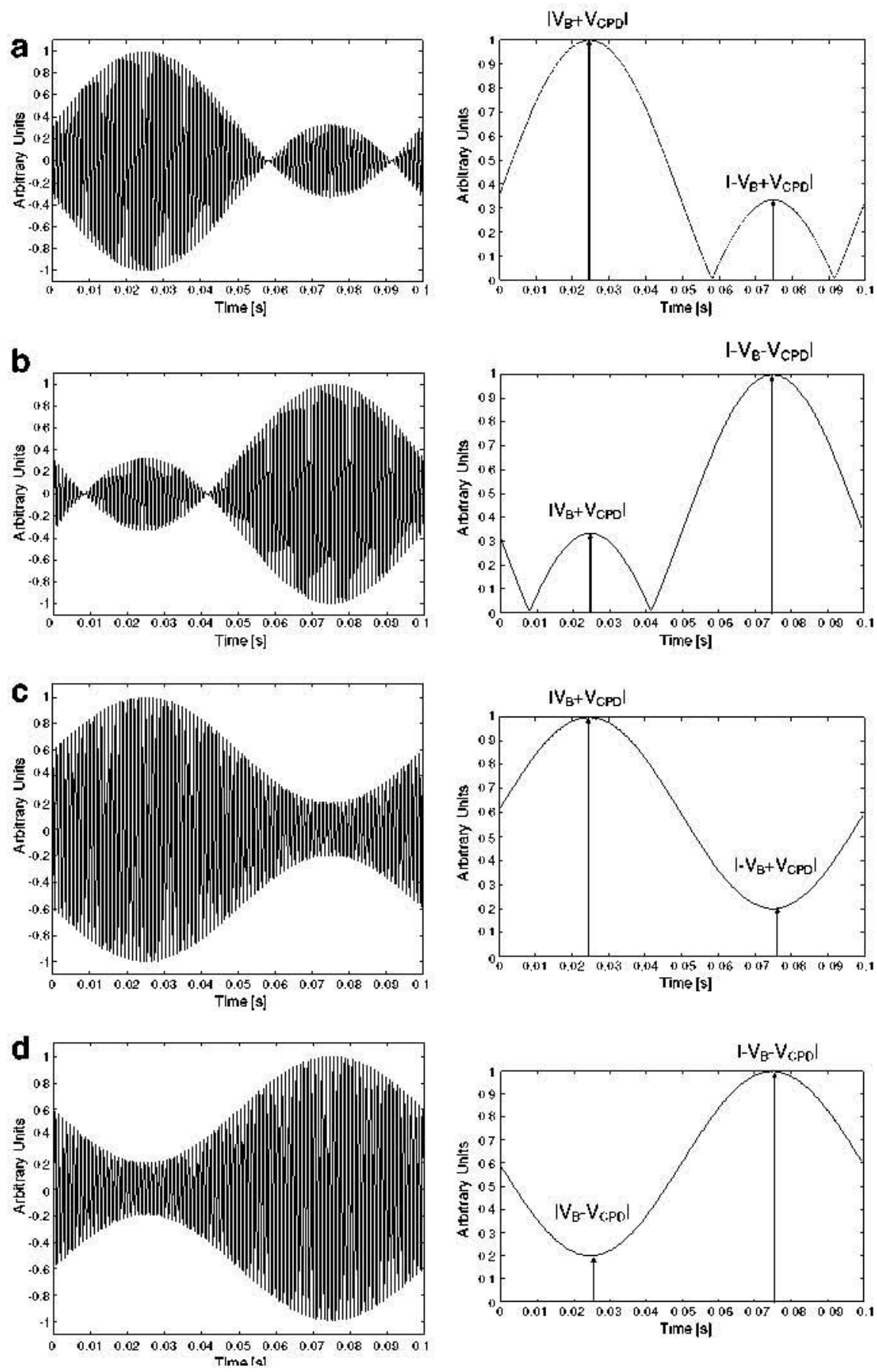

Fig. 3. Modulated KP signals (left) and processed signals (right) for: (a) Case 1, (b) Case 2, (c) Case 3, and (d) Case 4.

Height control is achieved by a combination of the double magnetic coils and a stepping positioner on which the KP head assembly is mounted. Fine height control of the head is accomplished by adding a DC voltage to the AC coil voltage. If the DC voltage on the coils exceeds a set limit, the distance is compensated by stepping the positioner motor. Furthermore, a fast Fourier transform (FFT) of the signal is performed during the measurement. A single peak in the FFT signal indicates that the KP needle is freely vibrating, but the appearance of multiple peaks in the FFT signal indicates that the KP needle is contacting the sample surface, in which 
case the needle is retracted from the surface.

The electrochemical control of potential occurs by passing a current to the CE, as in design A. However, in design $\mathrm{B}$, an AC backing potential is applied between WE and ground, so the whole electrochemical system floats with this backing potential. The right side of Fig. 2 shows some of the system used to control the potential. An optical isolator separates the ground for the two systems. An integrator is used to fix the potential as in design A. The input signal to the integrator is switched on when the difference between the measured potential and the desired potential is greater than some set value. The polarity and the magnitude of the integrator input signal determine the direction and rate of change of the potential control. When the switch is open, the potential is maintained by the capacitor in the integrator circuit. The desired scan rate is set and the step size and interval are determined by the stabilization time at a given potential. When the measured potential is sufficiently close to the set potential, the current, potential, and height are recorded into one data set and the next value of set potential is determined based on the scan rate. A continual record of current, potential and height is recorded at $1 \mathrm{~Hz}$ into a second data set for diagnostic purposes.

\section{Experimental}

Electrochemical measurements were made with both design A and design B KPPs. The samples for both were Type 304 stainless steel (SS) wires of $1 \mathrm{~mm}$ diameter surrounded by an Inconel 600 ring CE with 3 and $6 \mathrm{~mm}$ inner and outer diameter, respectively, and embedded in epoxy, Fig. 4. The samples were polished to 600 grit, resulting in a coplanar disk WE and ring $\mathrm{CE}$. The sample assembly was placed in the stainless steel KP chamber, which was purged continuously with humidified air to maintain the relative humidity (RH) at about $95 \%$. A small amount of $0.7 \mathrm{M} \mathrm{NaCl}$ solution was spread over the surface of the $\mathrm{CE}$ and WE. This solution was allowed to equilibrate with the $95 \% \mathrm{RH}$ air, resulting in a chloride concentration of about $1 \mathrm{M}$ [16]. The final thickness of the layer depended on the initial thickness and concentration, and was measured using a sharp PtIr wire. The potential between the wire and the WE was monitored as the wire was lowered (using a micrometer dial gauge in design A and the stepper motor in design B) until it touched first the solution surface and then the WE surface. Electrolyte was also banked around the periphery of the epoxy mount to dissipate charge on the polymer surface.

The KP needle was calibrated using $\mathrm{Cu} / \mathrm{CuSO} 4$ and the open circuit potential (OCP) of the WE was determined by measuring VCPD. Potential scanning was then initiated starting at the OCP and scanning either in the anodic or cathodic direction. With the design A instrument, $10 \mathrm{mV}$ steps were taken every $10 \mathrm{~s}$ in the anodic direction, and $10 \mathrm{mV}$ steps were taken every 30 $\mathrm{s}$ in the cathodic direction. For the anodic scan, the scan direction was reversed after pitting initiated. With the design $\mathrm{B}$ instrument, the scan rate was set at $1 \mathrm{mV} / \mathrm{s}$, but the effective scan rate was about $0.7 \mathrm{mV} / \mathrm{s}$ owing to stability issues. 


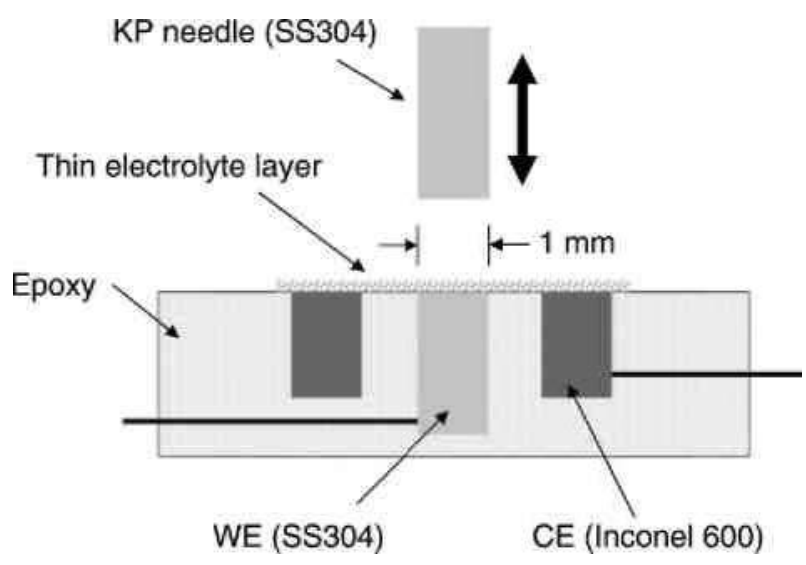

Fig. 4. Schematic cross-section of KP needle and sample including SS304 WE and Inconel 600 CE embedded in epoxy.

Dry-out experiments were performed with the design B KPP. In this experiment, a film of $0.5 \mathrm{M} \mathrm{MgCl} 2$ was applied to the 304L electrode assembly. After calibration, air with $33 \% \mathrm{RH}$ (created by equilibrium with saturated $\mathrm{MgCl}$ ) was passed through the chamber, resulting in slow evaporation of water from the electrolyte. As the water evaporated, the chloride concentration increased. After reaching a critical chloride concentration, pitting will initiate and the OCP will drop [15]. This phenomenon was tracked with the KPP by monitoring the OCP and solution layer thickness with time.

\section{Results and discussion}

\subsection{Cathodic polarization curves}

Fig. 5 shows some cathodic polarization curves for Type $304 \mathrm{SS}$ under $1 \mathrm{M} \mathrm{NaCl}$ solution layers of different thickness using the type-A KPP. Almost identical curves were observed using the type-B KPP for the same experiment. A limiting current region associated with transport of dissolved oxygen across the thin solution layer is clearly visible. At more negative potentials the current increased again owing to hydrogen evolution. The limiting current density values, $i \mathrm{lim}$, determined for a number of experiments are shown as a function of inverse solution thickness, $d$ soln, in Fig. 6. Also shown is a line with slope of 1, which is the expected relation between $i$ lim and inverse diffusion layer thickness in a double logarithmic plot:

$$
i_{\lim }=\frac{n F D C}{d}
$$

where $n$ is the number of electrons in the reaction, $F$ is the Faraday constant, $D$ is oxygen diffusivity, $C$ is oxygen concentration in equilibrium with air, and $d$ is diffusion layer thickness. A region is observed where the dependence of $i$ lim on $d$ soln- 1 is close to the expected relation. Furthermore, this plot reveals that $i$ lim for oxygen reduction depends on the $d$ soln only over a limited range, in agreement with the results of others [10,13]. As described above, convection controls the diffusion layer thickness in thick electrolyte layers and the transfer of oxygen across the air/solution interface is rate limiting for very thin layers. Assuming four-electron reduction of oxygen, oxygen diffusivity of $2.2 \times 10-5 \mathrm{~cm} 2 / \mathrm{s}$ [19], and oxygen concentration in equilibrium with air of 2.45 x 10-4 M [5], $n F D C$ should be 


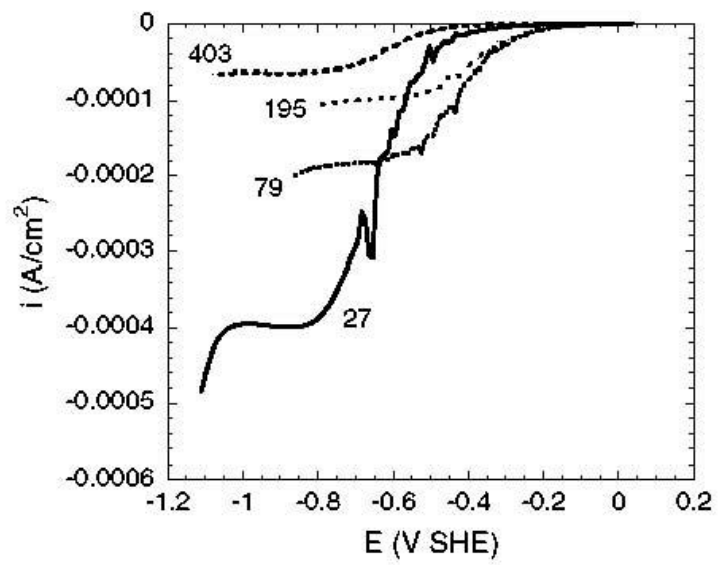

Fig. 5. Cathodic polarization curves for different solution layer thicknesses of $1 \mathrm{M} \mathrm{NaCl}$ on SS304. Numbers indicate layer thickness in $\mu \mathrm{m}$.

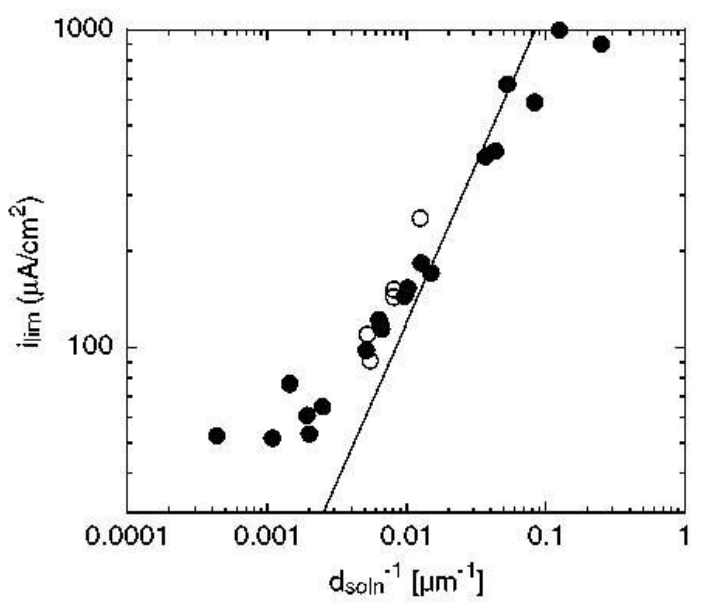

Fig. 6. Limiting current density as a function of inverse solution layer thickness. Filled and open symbols represent data collected with Design A and Design B KPPs, respectively. The line has a slope of 1.

$2.1 \times 10-6 \mathrm{~A} / \mathrm{cm}$. The intercept of the line in Fig. 6 indicates a measured value of $1.1 \times 10-6$ $\mathrm{A} / \mathrm{cm}$. Therefore the data are reasonable, indicating that the design A KPP system is capable of controlling potential and measuring polarization curves properly. The open symbols in Fig. 6 represent limiting current densities from experiments performed with the type-B KPP. They essentially overlie the other data, indicating that this instrument also can effectively control potential.

\subsection{Anodic polarization curves}

Three different cyclic polarization curves from experiments with the type-A KPP on Type 304SS in $1 \mathrm{M} \mathrm{NaCl}$ are shown in Fig. 7. The electrodes were spontaneously passive and then exhibited rapid increases in current associated with breakdown of the passive film and initiation of pitting corrosion. Fig. 8 shows the surface of one-electrode after the experiment with clear evidence of pits. Fig. 9 is a compilation of pitting potentials measured under solution layers of thickness ranging from 40 to $280 \mu \mathrm{m}$. At least over this range, no evidence of a dependence of pitting 
potential on solution layer thickness was evident. The spread of breakdown potentials measured on the same electrode configuration in a bulk solution of $1 \mathrm{M} \mathrm{NaCl}$ is also shown in Fig. 9. There is some indication that the thin film solution breakdown potentials are higher than those in bulk solutions, but there is no clear trend.

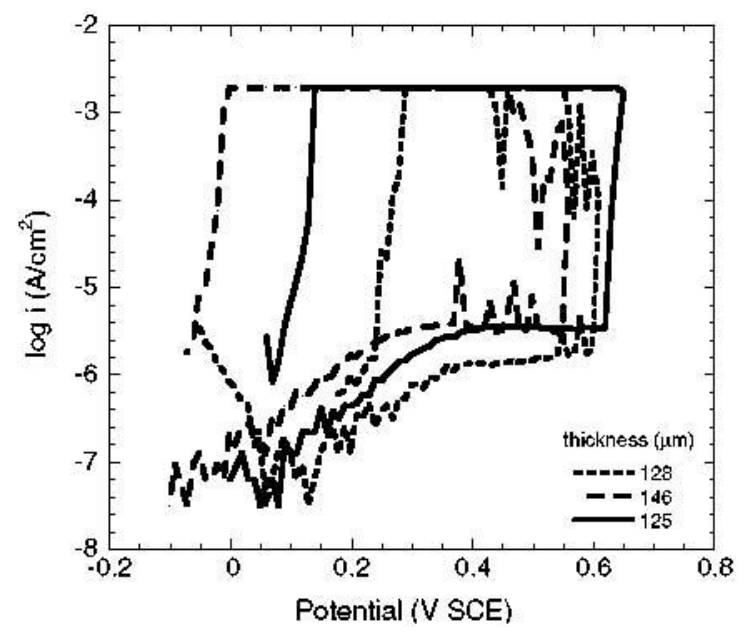

Fig. 7. Three anodic cyclic polarization curves for Type 304SS under thin layer of $1 \mathrm{M} \mathrm{NaCl}$.

The maximum current available from the integrator is $15 \mu \mathrm{A}$, which is equivalent to 1.9 $\mathrm{mA} / \mathrm{cm} 2$ for a $1 \mathrm{~mm}$ diameter electrode. This current limitation is clearly evident on the backscan portion of the cyclic curves. However, at some point during the backscan, the current dropped precipitously, associated with repassivation of the pits on the electrode surface. There was also no trend of repassivation potential with solution thickness.

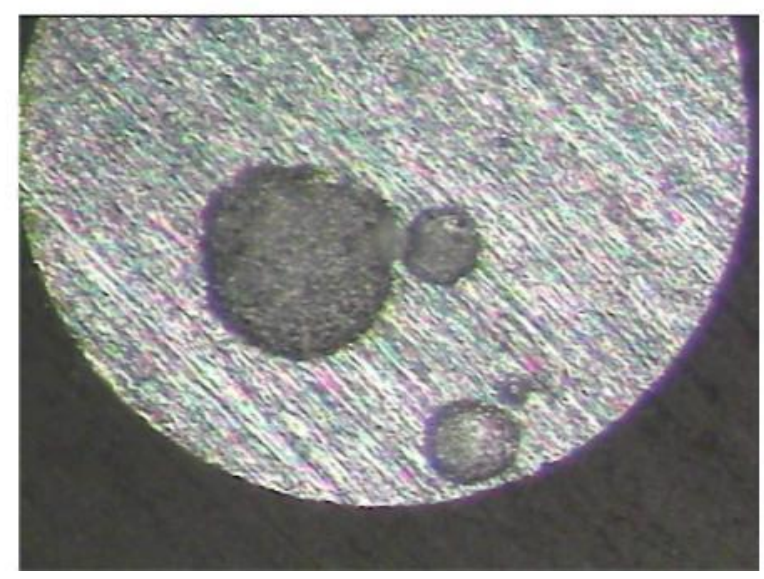

Fig. 8. Optical image of working electrode surface after anodic cyclic polarization experiment. Diameter of working electrode is $1 \mathrm{~mm}$. 


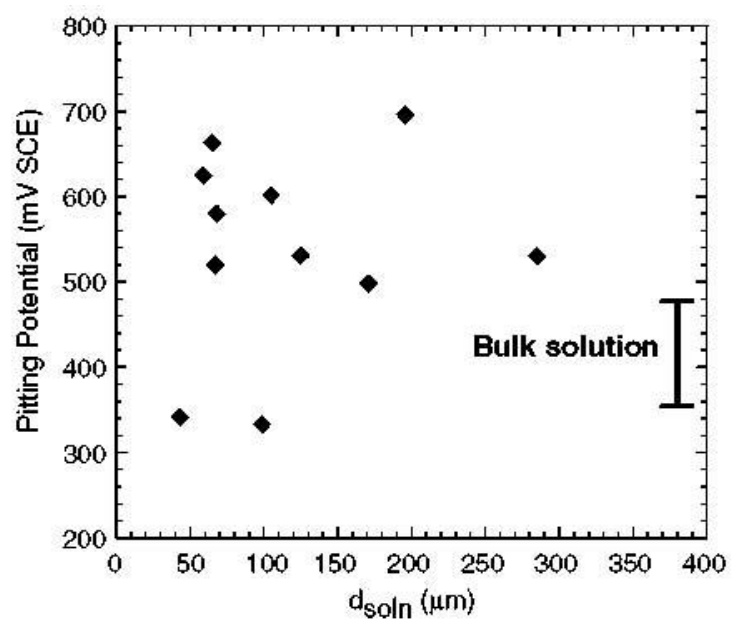

Fig. 9. Compilation of pitting potentials for a number of experiments under thin solution layer and the range of pitting potentials observed for the same sample in bulk solution.

An interesting phenomenon was observed during the cyclic polarization measurement, as shown in Fig. 10, which is a series of images of the tip and electrode. Fig. 10a is an image taken prior to the initiation of pitting corrosion. The KP needle is seen extending downward from the top of the image; the image is tilted with respect to vertical, so the needle appears to be at an angle. The sample is at the bottom; the WE is seen as the smallest circle. The dark ring around this circle is epoxy, as is the region far from the central WE. The CE is evident as a darkish ring between these two epoxy regions. The surface of the sample assembly is covered with a thin solution layer, and the bottom of the KP needle is reflected off of this layer. After initiation of pitting corrosion, the first clear observation was evolution of hydrogen gas at the inner diameter of the ring $\mathrm{CE}$ resulting in a thin ring of bubbles spraying off of the surface. Evidence for this ring is difficult to see in the images in Fig. 10, but a lightness is evident at the inner edge of the CE. With time of sustained pit growth, an apparent crease in the electrolyte formed just outside the WE and the thickness of the layer within this crease, i.e. on top of the WE, increased significantly, Fig. 10d-f. This was observed during every anodic polarization experiment after pit initiation. The solution thickness grew until eventually touching the vibrating needle, at which point the experiment ended because the KPP cannot maintain potential control with the needle immersed in the solution layer. The needle-tip/sample separation shown in Fig. 10a, about $1 \mathrm{~mm}$, was about 10 times larger than the typical separation to minimize the effect of this phenomenon. The solution layer thickness increased from an initial value of tens of $\mu \mathrm{m}$ to the $\mathrm{mm}$ range within a few minutes of pit growth. Another interesting observation, which is not at all seen in Fig. 10, was bubble evolution in the enlarged solution drop on top of the WE. 

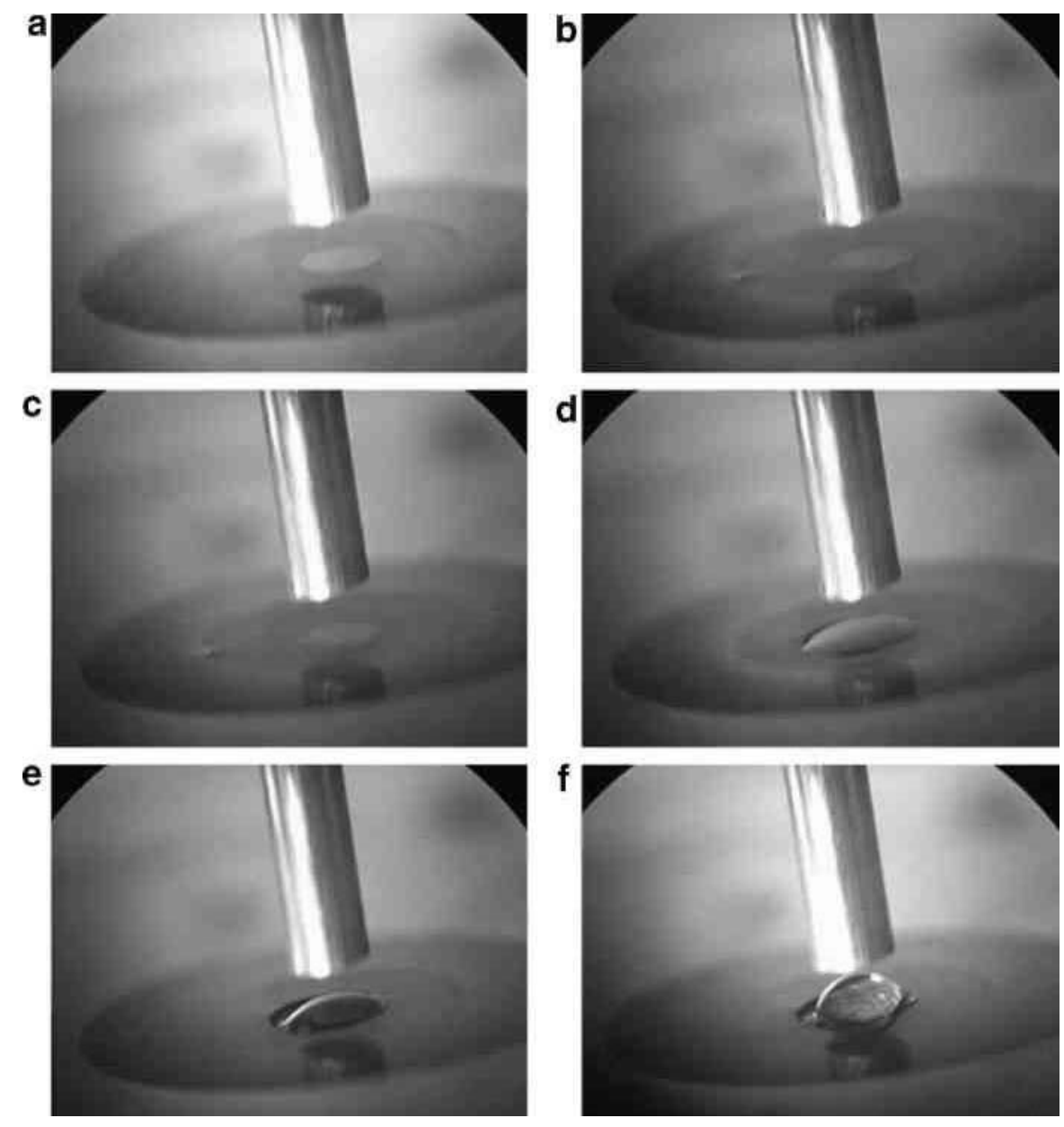

Fig. 10. Images of the needle and sample during pit growth under a thin solution layer showing evidence of a large increase in solution volume during pitting.

The huge increase in solution volume during pitting occurred because the thin solution layer, which was initially in equilibrium with the humid air environment $(95 \% \mathrm{RH})$, became concentrated with ions as a result of the pitting reaction. The highly concentrated solution layer was out of equilibrium with the humid air and it rapidly absorbed water from the air, which was replenished by continual flow of humid air through the chamber. In this fashion, the thin layer electrolyte essentially became a thick layer.

Fig. 11 shows images of a sample after anodic cyclic polarization immediately after removal from the chamber and after rinsing and drying. The apparent crease was associated with a thick precipitated corrosion product layer just beyond the edge of the WE. The separation of anode and cathode by a very thin solution layer resulted in a steep $\mathrm{pH}$ gradient and the formation of this precipitated product layer. The precipitated layer prevented transport of metal cations to the CE, but did not inhibit current flow, which continued during the experiment. The bubble evolution observed on the WE was apparently hydrogen evolution, which was promoted by the need to maintain charge neutrality in the thin solution layer.

It is as yet unclear whether the rapid absorption of water from the air into the thin solution layer will occur on naturally-formed pits or if it is a phenomenon that is only associated with pitting driven by the external potentiostat. 


\subsection{Open circuit dry-out measurements}

Fig. 12 shows the OCP and electrolyte layer thickness for a 304L sample as the electrolyte layer dried during exposure to a low RH environment. The initial thickness of the $0.5 \mathrm{M} \mathrm{MgCl} 2$ layer was $214 \mu \mathrm{m}$. Following calibration of the KP needle and measurement of the layer thickness, the $\mathrm{RH}$ in the chamber was decreased. These measurements were made with the type-B KPP, which as described above controls the separation of the KP needle and the electrolyte surface, thereby tracking of the change in the electrolyte layer thickness. The electrolyte layer thinned at a rate that was not constant, and reached $35 \mu \mathrm{m}$ after about $20 \mathrm{~min}$. The OCP was almost constant at the start of the experiment, and then increased before dropping precipitously at about 15 min. The form of this potential transient is identical to that observed using other techniques [15], and the potential drop was associated with the initiation and growth of pits. Pits were found on the sample surface after the experiment. This experiment illustrates the ability of the type-B potentiostat to control tip/sample distance and measure changes with time.
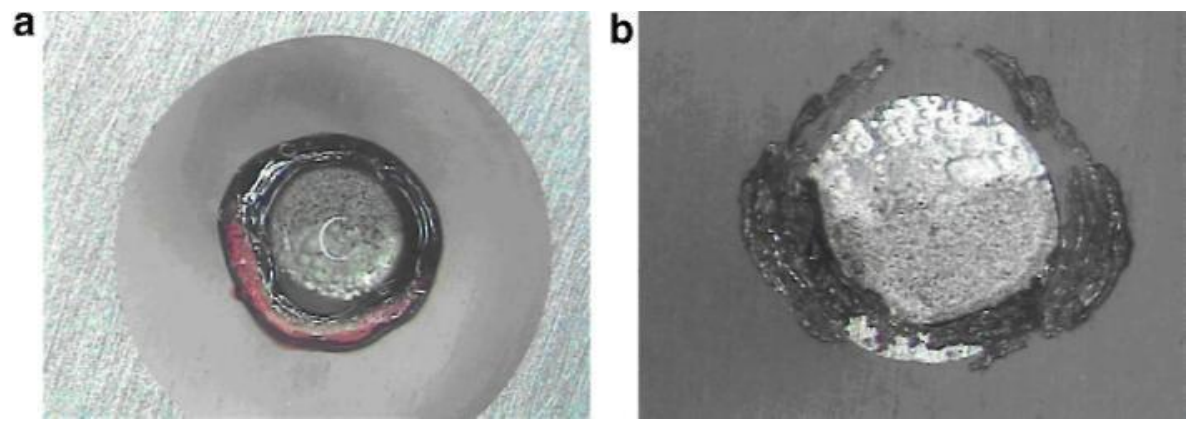

Fig. 11. Surface of a sample after anodic cyclic polarization: (a) immediately upon removal from the chamber and (b) after rinsing with water and drying.

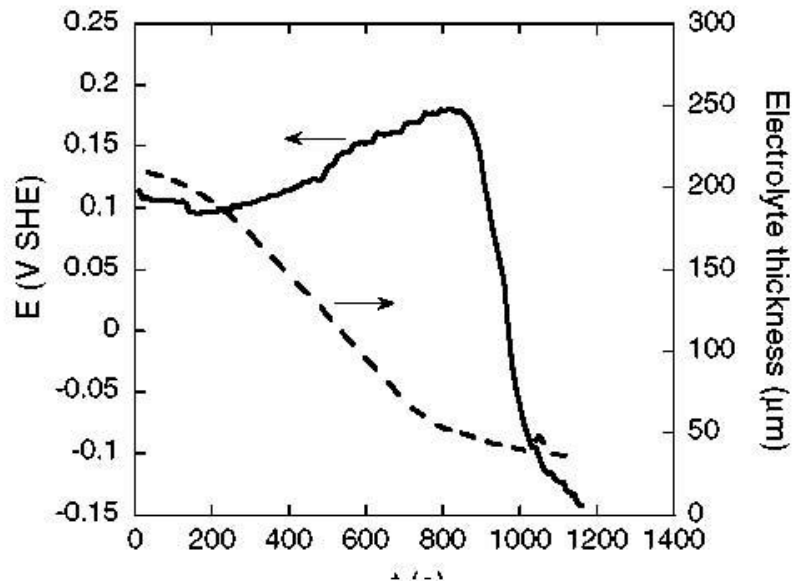

Fig. 12. OCP and electrolyte layer thickness as a function of time for a $0.5 \mathrm{M} \mathrm{MgCl} 2$ layer, initially $214 \mu \mathrm{m}$ thick, during drying in a low humidity.

\section{Conclusions}

The design and operation of two different Kelvin Probe Potentiostats are described and experiments with each are presented. The following was observed: 
1. Both KPP designs effectively controlled potential and allowed measurement of polarization curves under thin layers of electrolyte.

2. Cathodic polarization curves on Type 304L SS exhibited a limiting current density associated with oxygen reduction that varied with solution layer thickness over a finite range of thickness.

3. Pits were generated during anodic polarization of 304L under a thin electrolyte layer and the breakdown potential did not vary with layer thickness. However, after pit initiation, the increase in ionic strength of the thin layer resulted in a rapid absorption of water from the humid air environment.

4. The open circuit potential and chloride solution layer thickness were tracked with time during drying of the layer in a low humidity environment. Pit initiation occurred when the chloride concentration in the layer increased to a critical value.

\section{Acknowledgments}

This work was supported in part by the Office of Science and Technology and International (OST\&I), Office of Civilian Radioactive Waste Management (OCRWM), US Department of Energy (DOE). The work is carried out as part of the DOE Multi-University Corrosion Cooperative under Cooperative Agreement DE-FC2804RW12252. The views, opinions, findings, and conclusions or recommendations of authors expressed herein do not necessarily state or reflect those of the DOE/OCRWM/OST\&I. G.S.F. also received support for part of this work from the Alexander von Humboldt Foundation.

\section{References}

[1] F. Mansfeld, J.V. Kenkel, Corr. Sci. 16 (1976) 111.

[2] F. Mansfeld, J. Electrochem. Soc. 135 (1988) 1354.

[3] A. Cox, S.B. Lyon, Corr. Sci. 36 (1994) 1167.

[4] A. Cox, S.B. Lyon, Corr. Sci. 36 (1994) 1177.

[5] A. Cox, S.B. Lyon, Corr. Sci. 36 (1994) 1193.

[6] S.H. Zhang, S.B. Lyon, Corr. Sci. 36 (1994) 1289.

[7] S.H. Zhang, S.B. Lyon, Corr. Sci. 36 (1994) 1309.

[8] M. Stratmann, H. Streckel, Corr. Sci. 30 (1990) 681.

[9] M. Stratmann, H. Streckel, Corr. Sci. 30 (1990) 697.

[10] M. Stratmann, H. Streckel, K.T. Kim, S. Crockett, Corr. Sci. 30 (1990) 715.

[11] A. Nishikata, Y. Ishihara, T. Tsuru, Corr. Sci. 37 (1995) 897.

[12] A. Nishikata, Y. Yamashita, H. Katayama, T. Tsuru, A. Usami, K. Tanabe, H. Mabuchi, Corr. Sci. 37 (1995) 2059.

[13] A. Nishikata, Y. Ishihara, Y. Hayashi, T. Tsuru, J. Electrochem. Soc. 144 (1997) 244.

[14] R.P. Vera Cruz, A. Nishikata, T. Tsuru, Corr. Sci. 38 (1996) 1397.

[15] R.P. Vera Cruz, A. Nishikata, T. Tsuru, Corr. Sci. 40 (1998) 125.

[16] Y. Tsutsumi, A. Nishikata, T. Tsuru, J. Electrochem. Soc. 152 (2005) B358.

[17] M. Rohwerder, P. Leblanc, G.S. Frankel, M. Stratmann, Application of scanning Kelvin Probe in corrosion science, in: P. Marcus, F. Mansfeld (Eds.), Analytical Methods in Corrosion Science and Engineering, CRC Press LLC, 2006, pp. 605-648.

[18] I.D. Baikie, S. Mackenzie, P.J.Z. Estrup, J.A. Meyer, Rev. Sci. Instrum. 62 (1991) 1326.

[19] B. Case, Electrochim. Acta 18 (1973) 293. 